\title{
Ultra-low power wearables
}

\author{
B. D. Parameshachari ${ }^{1}$. Álvaro Rocha ${ }^{2} \cdot$ Chun Che Lance Fung ${ }^{3}$
}

(C) The Author(s), under exclusive licence to Springer-Verlag London Ltd., part of Springer Nature 2021

\section{Introduction and motivation}

The applications related to ultra-low power wearables like internet of things, robotics, intrusion detection, and image security have become a major computing paradigm and the latest disruptive technology after artificial intelligence. Ability of ultra-low power wearables to connect with other systems and applications from low to high level has prompted unprecedented array of applications in all fields of human activity from science, engineering, health, leisure and everyday life, etc. The technological growth in ultra-low power wearables is going hand-by-hand with enormous research efforts by an ever increasing community in a fascinating multidisciplinary field.

The motivation of this special issue is to offer an essential guide to the readers on the applications: internet of things security, wireless sensor network, image processing, intrusion detection, antenna, optimization, and cryptography. The accepted manuscripts are used as a reference text for graduate and undergraduate studies. Hence, the manuscripts are written in plain and easy to follow language and explain every main concept the first time it appears, helping readers with no prior background in the field. It is a "must-read" guide to the subject matter.

This special issue intends to provide a platform for researchers to share innovative work in many applications like internet of things security, wireless sensor network,

\footnotetext{
B. D. Parameshachari

paramesh@gsss.edu.in

Álvaro Rocha

amr@iseg.ulisboa.pt

Chun Che Lance Fung

L.Fung@ murdoch.edu.au

1 GSSS Institute of Engineering \& Technology for Women, Mysuru, Karnataka, India

2 ISEG, University of Lisbon, Lisbon, Portugal

3 College of Science, Health, Engineering and Education, Murdoch University, Perth, Australia
}

image processing, intrusion detection, antenna, optimization, and cryptography. After a double-blinded peer-review procedure, 14 papers have been accepted and included in this special issue, which contains different methods to solve different kinds of complex problems.

\section{Submission and summary of contributions}

In this issue, 14 manuscripts were accepted, and we believe that the works presented here will help researchers to do further analysis on the applications: internet of things security, wireless sensor network, image processing, intrusion detection, optimization, cryptography, and antenna. Firstly, we have a series of articles related to internet of things; Zhong et al. [1] introduced a new internet-based telemedicine system that assists patients to get health advice from doctors at home. The developed system significantly improves the throughput and avoids the repeated usage of the similar nodes compared to the traditional systems. In this manuscript, a more comprehensive telemedicine system was introduced, which effectively improves doctor's work efficiency and enables doctors and patients to communicate in real time. Xu and Wang [2] utilized a rapid search random tree method to remove obstacles in motion planning of the manipulator. The manipulator replaces human work in harsh environment such as radioactive, high pressure, flammable, high temperature, and explosive. The proposed rapid search random tree method significantly avoids collisions and quickly plans a non-collision path. This work is mainly used in the handling scenarios of robotic arms. Swamy and Alex [3] implemented a new reconfigurable filter bank using a parallel structure for reducing the delay in hearing aid applications with internet of things. The developed structure was validated with five dissimilar audiograms having mild conductive hearing loss, mild hearing loss at a middle frequency, mild hearing loss at high frequency, mild hearing loss at low frequency, and moderate hearing loss at high frequency. The experimental results showed that the developed structure achieved better matching between frequency 
response and audiogram of hearing. In this manuscript, the internet of things was utilized to select the sub-bands and to communicate the gain values to the hearing aid through a mobile application. Kalpana and Annadurai [4] introduced a new energy-efficient system of internet of things enabled body area networks. In this manuscript, an extreme learning machine was combined with the fog nodes to achieve low power network with high performance.

A few manuscripts related to "optimization" are summarized as follows: Kumar and Rajkumar [5] developed an optimized fuzzy logic social spider optimization algorithm for improving energy-efficient management to find software requirement prioritization. The recommendation of quality software development makes better device driven support in smart devices. Abid et al. [6] presented an optimized homomorphic encryption Chinese remainder theorem with a Rivest-Shamir-Adleman algorithm for an efficient and secure communication in the current digital world. Correspondingly, the seventh article "Achieving generalization of deep learning models in a quick way by adapting T-HTR learning rate scheduler" by Vidyabharathi et al. [7] implemented a new HTR learning rate scheduler (toggle between hyperbolic tangent decay and triangular mode with restarts) in order to identify an optimal learning rate in deep neural network. Compared to the traditional methods, the proposed learning rate scheduler consumed minimal time for every epoch in many cases. Further, a couple of manuscripts related to the topic "intrusion detection" are summarized as follows: Jothi and Pushpalatha [8] presented a whale integrated long short-term memory network for an effective intrusion detection. The developed network exhibited effective performance in differentiating the malicious nodes from the internet of things network and in detecting the network attacks. Though the simulation result showed that the developed network achieved accurate intrusion detection with limited predicting time, Sivanantham et al. [9] presented a rule precision index classifier to classify attack and non-attack internet of things network. The developed rule precision index classifier was built in WEKA tool, and its performance was evaluated on several intrusion datasets.

A manuscript on a topic "Communication antenna" is summarized as follows: Subramani et al. [10] analyzed antenna S11 variation (AS11V) with harmonic suppression for improving the communication process in medical applications. In this manuscript, an optimized recurrent neural network was used for processing the wearable antennabased collected devices. The eleventh paper "Feature-based autonomous target recognition and grasping of industrial robots" by Ruan et al. [11] analyzed the autonomous target recognition and grasping of industrial robot-based machine vision and deep learning techniques. Aruna and Usha [12] introduced a new dynamic substitution boxes, which were developed based on hybrid logistic Henon map with the deoxyribonucleic acid coding systems for improving the image security against the side channel attacks. The introduced system uses the 3D hybrid chaotic maps for increasing the key space and uses the deoxyribonucleic acid pattern of operation for enduring more security during transmission. Lastly, a couple of papers on the application "wireless sensor network" are summarized as follows: Sankayya et al. [13] have developed a novel protocol for minimizing the delay and maximizing the spectrum allocation. The developed protocol was implemented in real-time traffic monitoring application by using network simulator for estimating the quality of service. Yan et al. [14] simulated experiment with 30 drivers using a driving simulator for collecting the multi-sensor data of vehicle, human, and road condition. The driving risk status was classified into three states such as incident, near crash, and crash based on the playback system of the driving simulator.

Acknowledgements The guest editors are thankful to all reviewers for their efforts during reviewing the manuscripts. A special thank Editorin-chief Prof. Peter Thomas for his supportive guidance throughout the process.

\section{References}

1. Zhong Y, Xu Z, Cao L (2021) Intelligent IoT-based telemedicine systems implement for smart medical treatment. Pers Ubiquit Comput: 1-11. https://doi.org/10.1007/s00779-021-01633-1

2. Xu K, Wang Z (2021) Planning and control of mobile manipulator's operation on objects with restricted motion in intelligent environment. Pers Ubiquit Comput: 1-11. https://doi.org/10.1007/ s00779-021-01594-5

3. Swamy KA, Alex ZC (2021) Efficient low delay reconfigurable filter bank using parallel structure for hearing aid applications with IoT. Pers Ubiquit Comput: 1-14. https://doi.org/10.1007/ s00779-021-01600-w

4. Kalpana S, Annadurai C (2021) A novel energy efficient Fox-Ban architecture for body area networks. Pers Ubiquit Comput. https:// doi.org/10.1007/s00779-021-01652-y

5. Kumar KJ, Rajkumar N (2021) Improving energy-efficient management for identifying software requirement prioritization based on optimized fuzzy logic social spider optimization. Pers Ubiquit Comput: 1-10. https://doi.org/10.1007/s00779-021-01617-1

6. Abid R, Iwendi C, Javed AR, Rizwan M, Jalil Z, Anajemba JH, Biamba C (2021) An optimised homomorphic CRT-RSA algorithm for secure and efficient communication. Pers Ubiquit Comput: 1-14. https://doi.org/10.1007/s00779-021-01607-3

7. Vidyabharathi D, Mohanraj V, Kumar JS, Suresh Y (2021) Achieving generalization of deep learning models in a quick way by adapting T-HTR learning rate scheduler. Pers Ubiquit Comput: 1-19. https://doi.org/10.1007/s00779-021-01587-4

8. Jothi B, Pushpalatha M (2021) WILS-TRS-a novel optimized deep learning based intrusion detection framework for IoT networks. Pers Ubiquit Comput: 1-17. https://doi.org/10.1007/ s00779-021-01578-5

9. Sivanantham S, Mohanraj V, Suresh Y, Senthilkumar J (2021) Rule precision index classifier: an associative classifier with a 
novel pruning measure for intrusion detection. Pers Ubiquit Comput: 1-9. https://doi.org/10.1007/s00779-021-01599-0

10. Subramani P, Al-Turjman F, Kumar R, Kannan A, Loganthan A (2021) Improving medical communication process using recurrent networks and wearable antenna s11 variation with harmonic suppressions. Pers Ubiquit Comput: 1-13. https://doi.org/10.1007/ s00779-021-01526-3

11. Ruan D, Zhang W, Qian D (2021) Feature-based autonomous target recognition and grasping of industrial robots. Pers Ubiquit Comput: 1-13. https://doi.org/10.1007/s00779-021-01589-2

12. Aruna S, Usha G (2021) S-DAC: A novel dynamic substitution boxes using hybrid chaotic system and deoxyribonuceic Acid (DNA) coding for counterfeiting side-channel attacks. Pers Ubiquit Comput: 1-14. https://doi.org/10.1007/s00779-021-01579-4
13. Sankayya M, kumar Sakthivel R, Gayathri N, Al-Turjman F (2021) Wireless sensor network-based delay minimization framework for IoT applications. Pers Ubiquit Comput: 1-9. https://doi. org/10.1007/s00779-020-01517-w

14. Yan L, Gong Y, Chen Z, Li Z, Guo J (2021) Automatic identification method for driving risk status based on multi-sensor data. Pers Ubiquit Comput: 1-17. https://doi.org/10.1007/ s00779-021-01580-x

Publisher's note Springer Nature remains neutral with regard to jurisdictional claims in published maps and institutional affiliations. 